\title{
ADOLESCENTS' DRINKING AND DRUNKENNESS MORE LIKELY IN ONE-PARENT FAMILIES AND DUE TO POOR COMMUNICATION WITH MOTHER
}

\author{
Zuzana Tomčíková', Zuzana Dankulincová Veselská1,, Andrea Madarasová Gecková1, 2, ${ }^{2}$, Jitse P. van Dijk, ${ }^{1,4}$, \\ Sijmen A. Reijneveld ${ }^{4}$ \\ ${ }^{1}$ Graduate School Košice Institute for Society and Health, Medical Faculty, P. J. Šafárik University, Košice, Slovakia \\ ${ }^{2}$ Health Psychology Unit, Institute of Public Health, Medical Faculty, P. J. Šafárik University, Košice, Slovakia \\ ${ }^{3}$ Olomouc University Society and Health Institute, Palacký University in Olomouc, Czech Republic \\ ${ }^{4}$ Department of Social Medicine, University Medical Centre Groningen, University of Groningen, Groningen, Netherlands
}

\section{SUMMARY}

Objectives: Alcohol use is a relatively common behaviour, particularly among adolescents, and has become a major public health concern. This study explores the associations between family composition, the quality of adolescents' communication with parents and adolescents' recent frequent alcohol drinking and lifetime drunkenness.

Methods: Data were obtained from the Slovak part of the 2005-2006 Health Behaviour in School-aged Children (HBSC) study. The sample consisted of 3,882 students $(46.3 \%$ males; mean age 13.3; \pm 1.6$)$. Data on drinking alcohol in the past week, lifetime drunkenness, communication and family composition were collected via anonymous questionnaires stratified for ages 11,13 and 15 years and following the methodology of the HBSC study.

Results: The results showed that living in an incomplete family increased the risk of frequent drinking and drunkenness among adolescents as well as a low quality of communication between mothers and their children. Risks were higher for drunkenness than for frequent alcohol use and strongly increased by age, with the communication with parents worsening at increasing age.

Conclusions: Our findings show the importance of the quality of communication between parents and adolescents in preventing the hazardous alcohol use among adolescents. Preventive interventions to reduce adolescents' use of alcohol should therefore also target the quality of communication in the family.

Key words: adolescents, alcohol drinking, drunkenness, family composition, communication with parents

Address for correspondence: Z. Dankulincová Veselská, P. J. Šafárik University, Medical Faculty, Institute of Public Health, Health Psychology Unit, Tr. SNP 1, 04011 Košice, Slovakia. E-mail: zuzana.veselska@upjs.sk

\section{INTRODUCTION}

Alcohol use, specifically drunkenness, is a relatively common behaviour, particularly among adolescents, and has become a major public health concern. According to the most recent Health Behaviour in School-aged Children (HBSC) study (1), Slovak children start drinking alcohol at a relatively early age: $9 \%$ of girls and $14 \%$ of boys at the age of 11 years reported drinking alcohol at least once a week, and this proportion increases with age. The age of the first experience with drunkenness is also relatively low - at 15 years $31 \%$ of girls and $39 \%$ of boys have already had the experience of being drunk. Most of these first experiences with alcohol take place at home; as it is a common part of any party to offer small alcoholic toasts to children and adolescents.

Among the wide range of factors that influence risky alcohol consumption, the family environment is one of the most significant $(2,3)$. It is the developmental context in which the most important basic values, attitudes and patterns of behaviour are formed. One of the crucial parts of family functioning is adequate communication between parents and their children, which has been shown to be an important protective factor (1). Good communication with parents is an indicator of social support from parents and of family connectedness (4). Poor parent-child communication was found to be associated with a higher prevalence of youth substance use $(1,5)$. Adolescence is a unique period regarding communication with parents - children speak less often with their parents about themselves and communication becomes generally more difficult (6). Typically, for both adolescent boys and girls communication with the mother is better than with the father (7).

Another important aspect of family life is family composition, which is defined in HBSC studies as a configuration of people living in one household. Basically, family composition consists of family members, their common relationships and their presence or absence. During the last decades, the family has undergone very important changes - inter alia, the number of one parent families and of families with step parents has increased. The former in particular represents a great risk regarding a child's development, as children and adolescents living in one-parent families are more often involved in risk behaviour, including alcohol use $(8,9)$. This might be due to reduced parental control (10), a reduced socioeconomic position, both of which often occur in one-parent families (11), or due to different parenting (e.g. parental warmth) (12). 
Based on theoretical and empirical findings, the aim of this study is to explore the association between family composition and the quality of the adolescent's communication with parents with adolescent frequent alcohol drinking and lifetime drunkenness, and differences regarding this by age and gender.

\section{MATERIALS AND METHODS}

\section{Participants and Procedure}

Data were obtained from the Slovak part of the 2005-2006 Health Behaviour in School-aged Children (HBSC) study, a multinational study that was conducted in collaboration with the World Health Organization (1). The Slovak sample consisted of 3,882 students ( $46.3 \%$ males) aged 10 to 16 (mean age $13.3 ; \pm 1.6$ ). Respondents were divided into three age categories - 11 years old $(\mathrm{N}=1,298 ; 46.8 \%$ males $), 13$ years old $(\mathrm{N}=1,327 ; 44.8 \%$ males), and 15 years old ( $\mathrm{N}=1,252 ; 47.2 \%$ males $)$. The students completed the questionnaire on a voluntary and anonymous basis without a teacher's presence in the classroom, according to the methodology of the HBSC-study.

\section{Measures}

\section{Frequent Alcohol Drinking}

Respondents were asked how often they had drunk five different types of alcoholic drinks (beer, wine, spirits, alcopops low-alcohol flavoured drinks, and other) in the past month, with possible responses never, rarely, every month, every week, every day. A dichotomized variable was constructed for the analysis never, rarely or monthly/every day or every week (= frequent alcohol drinking).

\section{Lifetime Drunkenness}

Respondents were asked whether they had ever, during their lifetime, had so much alcohol that they were "really drunk". Possible responses ranged from never to yes, more than 10 times. A dichotomized variable was constructed for the analysis - never/ at least once.

\section{Communication with Parents}

Respondents were asked how easy it is for them to talk to their mother and father, respectively, about "things that really bother you," with possible responses: very easy, easy, difficult, very difficult. A dichotomized variable was constructed for the analysis - easy/difficult.

\section{Family Composition}

To asses family composition the following indicator was used: "All families are different and we would like to know yours. Here is a list of some of the people that can make up a family. Please tick one box for each line to show if you live with the person or, if you don't live with them, how often you see them." In this particular study only the information on parents and stepparents was used. Respondents were divided into two groups $-\mathrm{a}$ complete family (living with two parents or step parents) and a single-parent family.

\section{Statistical Analyses}

All data were analyzed using SPSS, version 16. We first assessed the characteristics of the sample across the three age categories. Next, a binary logistic regression (enter method) was performed leading to odds ratios (OR) with associated $95 \%$ confidence intervals (CI). First, three models were constructed analyzing the associations with frequent alcohol drinking, and then the same analyses were performed for the associations with lifetime drunkenness. In the first model we assessed the crude effects of family composition, communication with father/mother, gender and age. In the second model we analyzed the association of the family composition, the quality of communication with each parent and gender with frequent alcohol drinking/drunkenness. In the last model, age was added to the previous variables. The share of missing values was approximately $18 \%$. To make sure that this amount of missing values would not affect the findings, all analyses were repeated with including the 'missing' category as a separate category. No differences were found regarding the findings.

\section{RESULTS}

A description of the sample and its characteristics can be found in Table 1.

Table 2 shows the results of the logistic regression analysis for the association of family composition, communication with father, communication with mother, gender, and age with frequent alcohol drinking among adolescents. The first model assessed the crude effects of all the mentioned variables. All of them were found to be associated with frequent alcohol drinking: living in an incomplete family, difficult communication with parents, male gender, and higher age increase the probability of frequent alcohol drinking among adolescents. In the next model we assessed the joint effects of family composition, communication with mother/ father and gender. Family composition and communication with mother continued to show a statistically significant association, but the communication with father did not. A significant effect of gender was found in this model: male gender increases the probability of frequent alcohol use. In the last step, age was added to the model. Family composition and gender were found to be significantly associated with alcohol drinking, but communication with parents was not. A significant effect of age was found in this model: the risk of frequent drinking increased with age.

Table 3 shows the results of the logistic regression analysis for the association of family composition, communication with father, communication with mother, gender, and age with lifetime drunkenness among adolescents. In the first model the crude effects of all mentioned variables were assessed. All of them were found to be associated with drunkenness: living in an incomplete family, difficult communication with parents, male gender and higher age increased the probability of drunkenness among adolescents. In the second model we assessed the joint effects of family composition, communication with mother/father and gender. All of them continued to show a statistically significant association. In the last model, age was added to the model. Family composition, communication with mother and gender remained significantly associated with drunkenness, but communication with father did not. A significant effect of age was found in this model: the risk of drunkenness increases with age. 
Table 1. Characteristics of the study sample by age and gender

\begin{tabular}{|c|c|c|c|c|c|c|c|}
\hline & & \multicolumn{2}{|c|}{11 years } & \multicolumn{2}{|c|}{13 years } & \multicolumn{2}{|c|}{15 years } \\
\hline & & Male & Female & Male & Female & Male & Female \\
\hline & & $N=608$ & $N=690$ & $N=595$ & $N=732$ & $N=591$ & $N=661$ \\
\hline Frequent alcohol drinking & yes & $13.8 \%$ & $9.1 \%$ & $16.3 \%$ & $12.2 \%$ & $33.5 \%$ & $21.7 \%$ \\
\hline Lifetime drunkenness & at least once & $11.6 \%$ & $9.2 \%$ & $30.7 \%$ & $26.3 \%$ & $58.3 \%$ & $48.1 \%$ \\
\hline Family composition & incomplete & $7.4 \%$ & $9.6 \%$ & $10.6 \%$ & $12.4 \%$ & $14.2 \%$ & $14.2 \%$ \\
\hline \multirow[t]{4}{*}{ Communication father } & very easy & $42.4 \%$ & $28.5 \%$ & $33.5 \%$ & $13.8 \%$ & $24.3 \%$ & $11.7 \%$ \\
\hline & easy & $39.4 \%$ & $43.9 \%$ & $43.7 \%$ & $41.9 \%$ & $42.9 \%$ & $37.8 \%$ \\
\hline & difficult & $14.0 \%$ & $18.5 \%$ & $16.9 \%$ & $31.4 \%$ & $22.3 \%$ & $30.9 \%$ \\
\hline & very difficult & $4.2 \%$ & $9.2 \%$ & $5.9 \%$ & $12.9 \%$ & $10.5 \%$ & $19.6 \%$ \\
\hline \multirow[t]{4}{*}{ Communication mother } & very easy & $53.5 \%$ & $55.1 \%$ & $46.4 \%$ & $40.0 \%$ & $35.2 \%$ & $33.0 \%$ \\
\hline & easy & $36.8 \%$ & $33.7 \%$ & $10.4 \%$ & $46.5 \%$ & $45.2 \%$ & $47.7 \%$ \\
\hline & difficult & $7.6 \%$ & $10.0 \%$ & $10.5 \%$ & $11.5 \%$ & $16.4 \%$ & $15.3 \%$ \\
\hline & very difficult & $2.1 \%$ & $1.3 \%$ & $2.7 \%$ & $2.0 \%$ & $3.1 \%$ & $4.0 \%$ \\
\hline
\end{tabular}

Table 2. Binary logistic regression estimates for the effect of family composition, communication with mother, communication with father, gender, age, and family affluence on frequent alcohol drinking

\begin{tabular}{|c|c|c|c|c|c|}
\hline & & \multicolumn{4}{|c|}{$\begin{array}{l}\text { Frequent alcohol drinking } \\
\text { Odds ratio ( } 95 \% \text { confidence interval) }\end{array}$} \\
\hline & & Model 1 & Model 2 & Model 3 & Model 4 \\
\hline & & crude effects & adjusted effects $\#$ & adjusted effects ${ }^{\#}$ & adjusted effects ${ }^{\#}$ \\
\hline \multirow[t]{2}{*}{ Family composition } & complete & 1 (Ref.) ${ }^{* * *}$ & 1 (Ref.) ${ }^{* *}$ & 1 (Ref.) * & 1 (Ref.) * \\
\hline & incomplete & $1.88(1.52-2.32)$ & $1.63(1.17-2.28)$ & $1.51(1.08-2.13)$ & $1.52(1.08-2.13)$ \\
\hline \multirow[t]{2}{*}{ Communication father } & easy & 1 (Ref.) ${ }^{* * *}$ & 1 (Ref.) ${ }^{\text {ns }}$ & 1 (Ref.) $)^{\text {ns }}$ & 1(Ref.) ns \\
\hline & difficult & $1.46(1.25-1.71)$ & $1.90(0.94-1.49)$ & $1.04(0.83-1.32)$ & $1.04(0.82-1.31)$ \\
\hline \multirow[t]{2}{*}{ Communication mother } & easy & 1 (Ref.) ${ }^{* * *}$ & 1 (Ref.) * & 1 (Ref.) ${ }^{\text {ns }}$ & 1 (Ref.) ${ }^{\text {ns }}$ \\
\hline & difficult & $1.67(1.37-2.04)$ & $1.34(1.01-1.77)$ & $1.25(0.94-1.66)$ & $1.24(0.93-1.65)$ \\
\hline \multirow[t]{2}{*}{ Gender } & female & 1 (Ref.) ${ }^{* * *}$ & 1 (Ref.) $)^{* * *}$ & 1 (Ref.) ${ }^{* * *}$ & 1 (Ref.) ${ }^{* * *}$ \\
\hline & male & $1.32(1.14-1.51)$ & $1.64(1.34-2.01)$ & $1.63(1.33-2.01)$ & $1.62(1.32-1.99)$ \\
\hline \multirow[t]{3}{*}{ Age category } & 11 years & 1 (Ref.) ${ }^{* * *}$ & & 1 (Ref.) ${ }^{* * *}$ & 1 (Ref.) ${ }^{* * *}$ \\
\hline & 13 years & $3.43(2.74-4.30)$ & & $1.31(0.98-1.75)$ & $1.31(0.98-1.75)$ \\
\hline & 15 years & $9.76(7.84-2.16)$ & & $3.03(2.32-3.94)$ & $3.05(2.34-3.97)$ \\
\hline \multirow[t]{3}{*}{ Family affluence } & high & 1 (Ref.) ${ }^{\text {ns }}$ & & & 1 (Ref.) ${ }^{\text {ns }}$ \\
\hline & medium & $0.83(0.63-1.08)$ & & & $0.81(0.60-1.10)$ \\
\hline & low & $0.89(0.68-1.15)$ & & & $0.92(0.70-1.24)$ \\
\hline
\end{tabular}

${ }^{*} p<0.05,{ }^{* *} p<0.01,{ }^{* * *} p<0.001 ; n s=$ not significant; Ref = reference category

\# The odds ratios for each variable have been adjusted for the effects of the other variables in the model

\section{DISCUSSION}

The current study explored the associations of family composition and communication with parents with frequent alcohol drinking (at least once a week) and lifetime drunkenness among adolescents. The study contributes to the knowledge by using two indicators of risky alcohol use. Both of these risky patterns are particularly important in adolescence. Frequent alcohol use in adolescence might be a predictor of alcohol problems in adulthood, leading not only to alcohol dependence, but also to chronic physical and mental health problems in later life (13). Adolescent drunkenness, on the other side, might lead directly to a serious intoxication and eventually to death. Living in a single-parent family increased the risk of both frequent alcohol drinking and drunkenness among adolescents, and this effect remained after adjustment for communication with parents, gender and age. Secondly, poor communication with parents increased the risk of frequent alcohol drinking and drunkenness among adolescents. Regarding frequent drinking, the association with communication with the father disappeared after adjustment for communication with the mother, family composition, gender, and age. The association with communication with the mother decreased in strength. 
Table 3. Binary logistic regression estimates for the effect of family composition, communication with mother, communication with father, gender, age, and family affluence on drunkenness

\begin{tabular}{|c|c|c|c|c|c|}
\hline & & \multicolumn{4}{|c|}{$\begin{array}{c}\text { Drunkenness } \\
\text { Odds ratio ( } 95 \% \text { confidence interval) }\end{array}$} \\
\hline & & Model 1 & Model 2 & Model 3 & Model 4 \\
\hline & & crude effects & adjusted effects ${ }^{\#}$ & adjusted effects ${ }^{\#}$ & adjusted effects ${ }^{\#}$ \\
\hline \multirow[t]{2}{*}{ Family composition } & complete & 1 (Ref.) ${ }^{* \star *}$ & 1 (Ref.) ${ }^{* * *}$ & 1 (Ref.) $)^{* * *}$ & $1(\text { Ref.) })^{* *}$ \\
\hline & incomplete & $1.88(1.52-2.32)$ & $2.36(1.78-3.14)$ & $2.23(1.64-3.04)$ & $2.25(1.65-3.06)$ \\
\hline \multirow[t]{2}{*}{ Communication father } & easy & 1 (Ref.) ${ }^{* * *}$ & 1 (Ref.) $)^{* * *}$ & 1 (Ref.) ns & 1 (Ref.) ${ }^{\text {ns }}$ \\
\hline & difficult & $1.46(1.25-1.71)$ & $1.41(1.18-1.70)$ & $1.15(0.94-1.40)$ & $1.15(0.94-1.40)$ \\
\hline \multirow[t]{2}{*}{ Communication mother } & easy & 1 (Ref.) ${ }^{* * *}$ & 1 (Ref.) $)^{* *}$ & 1 (Ref.) ${ }^{* *}$ & 1 (Ref.) ** \\
\hline & difficult & $1.67(1.37-2.04)$ & $1.44(1.14-1.82)$ & $1.36(1.06-1.75)$ & $1.36(1.06-1.75)$ \\
\hline \multirow[t]{2}{*}{ Gender } & female & 1 (Ref.) $)^{* * *}$ & 1 (Ref.) ${ }^{* * *}$ & 1 (Ref.) $)^{* * *}$ & 1 (Ref.) $)^{* * *}$ \\
\hline & male & $1.32(1.14-1.51)$ & $1.46(1.24-1.72)$ & $1.47(1.23-1.76)$ & $1.46(1.22-1.75)$ \\
\hline \multirow[t]{3}{*}{ Age category } & 11 years & 1 (Ref.) ${ }^{* * *}$ & & 1 (Ref.) ${ }^{* * *}$ & 1 (Ref.) ${ }^{* * *}$ \\
\hline & 13 years & $3.43(2.74-4.30)$ & & $3.57(2.74-4.64)$ & $3.57(2.74-4.64)$ \\
\hline & 15 years & $9.76(7.84-12.16)$ & & $9.80(7.58-12.66)$ & $9.83(7.61-12.71)$ \\
\hline \multirow[t]{3}{*}{ Family affluence } & high & 1 (Ref.) ns & & & 1 (Ref.) ns \\
\hline & medium & $0.97(0.78-1.21)$ & & & $0.87(0.67-1.13)$ \\
\hline & low & $0.96(0.77-.19)$ & & & $0.87(0.67-1.13)$ \\
\hline
\end{tabular}

${ }^{* *} p<0.01,{ }^{* * *} p<0.001 ;$ ns = not significant; Ref = reference category

\# The odds ratios for each variable have been adjusted for the effects of the other variables in the model

Regarding lifetime drunkenness, the same occurred but almost all associations are somewhat stronger. The associations found were not significantly modified by gender or age.

Our findings regarding the association of living in a singleparent family with both frequent alcohol use and drunkenness in adolescents confirms the findings of several previous studies (9, 11, 14). Adolescents living in single-parent families are at a higher risk of trying alcohol earlier and drinking more hazardously (15). This might be inter alia related to lowered parental control (10). When there is only one parent who has to perform the tasks of both parents, this could lead to decreased control of adolescent behaviour, thus opening up more opportunities for risk behaviour in general and for experimentation with alcohol in particular. Adequate parental control has been shown to have both a direct and indirect impact, through affecting associations with peers who drink, on adolescent behaviour regarding alcohol use (16).

Another main finding of our study is that when communication with parents is perceived as difficult, the risk of frequent drinking among adolescents and drunkenness increases, which is in line with certain other studies $(5,17)$. Good quality communication has been shown to act as a protective factor with regard to youth substance use (1). One possible pathway for this effect might be again via parental control, which is changing in this period of life. It becomes less about direct observation and more about communication between parents and the adolescent when compared to earlier years. Good quality communication might thus lead to effective parental control, and so to a decreased risk of substance use (18).

The fact that communication with parents was more strongly associated with lifetime drunkenness than with frequent drinking might be explained by the outcome measures themselves. To drink alcohol is relatively highly tolerated in the Slovak society, and as we have already mentioned, the first experiences with alcohol often take place at home in the presence of parents. On the other hand, getting drunk is not tolerated, particularly in this age. Communication with parents matters more from the perspective of being drunk, because drunkenness is considered to be more risky than only alcohol consumption. Another interesting aspect of our findings is the fact that communication with the mother is more strongly associated particularly with drunkenness than communication with the father and that age seems to play an important role in this aspect - with increasing age, communication with the father becomes less important regarding frequent alcohol drinking and drunkenness. The same holds true for communication with the mother, but only in the case of drunkenness.

The fact that age had a strong effect on both adolescent frequent drinking and adolescent drunkenness is not surprising; it is a well-known fact from the literature (1). With increasing age adolescents have more opportunities to experiment with alcohol because of their growing independence from their parents, and they spend an increased amount of time unsupervised outside the home $(19,20)$. In addition, the association between both outcome measures and communication with the father largely decreases if age is added to the model, so the age-effect might be explained also by poorer quality of communication of adolescents with the father in particular when they grow older.

The present study has several strengths and limitations. A first strength is the size of the study sample and its representativeness for the regions of Slovakia. Selection bias was unlikely due to the way the sample was drawn. Another strength of the study is the use of two indicators of alcohol use (frequent alcohol drinking and drunkenness). A main limitation of our study is that it relied on the 
self-reporting of respondents. However, the questionnaires were filled in anonymously, which has been shown to produce rather valid self-reports (21). Another limitation of the study is the lack of socioeconomic data, which would enable to frame the findings in a broader context. In addition, the cross-sectional design of our study might be considered as limitation as it makes it impossible to formulate conclusive statements about causality in our findings. Poor communication may be both the cause and the consequence of alcohol abuse of adolescents and/or their parents. Beside this, poor communication may be related to other risk factors currently not covered by presented study. Our findings therefore need to be confirmed in studies with a longitudinal design with more potential risk factors included in order to approach the studied topic in a more complex way. Finally, our study is based on a Slovakian sample. The explored associations may vary between different cultures and settings.

\section{CONCLUSION}

Our findings show the importance of the quality of communication between parents and adolescents in preventing the hazardous alcohol use among adolescents. At higher adolescent age, this communication tends to deteriorate, which is associated with higher risks. In contemporary society, when the rates of excessive drinking in the European Union are increasing, this issue requires attention. Our results show that particular groups (adolescents living in incomplete families) run a higher risk of both frequent alcohol use and drunkenness and thus need particular attention in prevention. In particular, drunkenness seems to be a serious problem in terms of the vulnerability against the risk factors as well as in terms of the acute health threat. The prevention efforts should therefore focus not only on abstinence, but also on harm reduction interventions in adolescents who already drink frequently. Since the design of this study was cross-sectional, the implication for further research might be to study longitudinal data to confirm the hypothesized causal mechanisms with regard to frequent alcohol use and drunkenness. Moreover, it would be of interest whether parental communication has similar effects in countries with a different political and economic history.

\section{Acknowledgements}

This work was supported by the Slovak Research and Development Agency under the contract No. APVV-0032-11. This work was also partially supported (30\%) by the Agency of the Slovak Ministry of Education for the Structural Funds of the EU under project ITMS: 26220120058. This article was supported by the project OP VK named Social determinants of health by socially and health disadvantaged and other groups of population (CZ.1.07/2.3.00/20.0063).

\section{Conflict of Interests}

None declared

\section{Ethical Recommendations}

The study was approved by the Ethics Committee of the Faculty of Medicine at the PJ Šafárik University in Košice.

\section{REFERENCES}

1. Currie C, Gabhainn SN, Godeau E, Roberts C, Smith R, Currie D, et al., editors. Inequalities in young people's health: Health Behaviour in School-aged Children (HBSC) international report from the 2005/2006 survey. Health policy for children and adolescents, no. 5. Copenhagen: WHO Regional Office for Europe; 2008.

2. Kuntsche EN, Kuendig H. What is worse? A hierarchy of family-related risk factors predicting alcohol use in adolescence. Subst Use Misuse. 2006;41(1):71-86.

3. Weinberg NZ, Rahdert E, Colliver JD, Glantz MD. Adolescent substance abuse: a review of the past 10 years. J Am Acad Child Adolesc Psychiatry. 1998 Mar;37(3):252-61.

4. Laursen B. Conflict and social interaction in adolescent relationships. J Res Adolesc. 1995;5(1):55-70.

5. Griffin KW, Botvin GJ, Scheier LM, Diaz T, Miller NL. Parenting practices as predictors of substance use, delinquency, and aggression among urban minority youth: moderating effects of family structure and gender. Psychol Addict Behav. 2000 Jun;14(2):174-84.

6. Barnes HL, Olson DH. Parent-adolescent communication and the circumplex model. Child Dev. 1985;56:438-47.

7. Noller P, Callan VJ. Understanding parent-adolescent interactions: Perceptions of family members and outsiders. Dev Psychol. 1988;24(5):707-14.

8. Hoffmann JP. Family structure, community context, and adolescent problem behaviors. J Youth Adolesc. 2006;35(6):867-80.

9. Tomcikova Z, Madarasova Geckova A, Orosova O, van Dijk JP, Reijneveld SA. Parental divorce and adolescent drunkenness: role of socioeconomic position,psychological well-being and social support. Eur Addict Res. 2009;15(4):202-8.

10. Marsden J, Boys A, Farrell M, Stillwell G, Hutchings K, Hillebrand J, et al. Personal and social correlates of alcohol consumption among midadolescents. Br J Dev Psychol. 2005;23(3):427-50.

11. Rodgers KB, Rose HA. Risk and resiliency factors among adolescents who experience marital transitions. J Marriage Fam. 2002;64(4):1024-37.

12. Broman CL, Li X, Reckase M. Family structure and mediators of adolescent drug use. J Fam Issues. 2008;29(12):1625-49.

13. Schmid B, Hohm E, Blomeyer D, Zimmermann US, Schmidt MH, Esser G, et al. Concurrent alcohol and tobacco use during early adolescence characterizes a group at risk. Alcohol Alcohol. 2007 MayJun;42(3):219-25.

14. Paxton RJ, Valois RF, Drane JW. Is there a relationship between family structure and substance use among public middle school students? J Child Fam Stud. 2007;16(5):593-605.

15. Kirby JB. From single-parent families to stepfamilies: is the transition associated with adolescent alcohol initiation? J Fam Issues. 2006;27(5):685711.

16. Freisthler B, Byrnes HF, Gruenewald PJ. Alcohol outlet density, parental monitoring, and adolescent deviance: a multilevel analysis. Child Youth Serv Rev. 2009 Mar;31(3):325-330.

17. Eickhoff C, Zinnecker J. Protection or risk? Family environments reflected in communication between parents and their children. Research and practice of health promotion, vol. 11. Cologne: Federal Centre for Health Education; 2000.

18. Clark DB, Kirisci L, Mezzich A, Chung T. Parental supervision and alcohol use in adolescence: developmentally specific interactions. J Dev Behav Pediatr. 2008 Aug;29(4):285-92.

19. Loukas A, Prelow HM. Externalizing and internalizing problems in lowincome Latino early adolescents: Risk, resource, and protective factors. J Early Adolesc. 2004;24(3):250-73.

20. Moreno C, Borup I, Smith B, Kuzman M, Massa M, Zaborskis A, et al. HBSC Research Protocol for 2005/06 Survey. Section 2, Scientific rationales for focus areas: Family Culture. 2005.

21. Del Boca FK, Noll JA. Truth or consequences: the validity of self-report data in health services research on addictions. Addiction. 2000 Nov;95 Suppl 3:S347-60. 